\title{
UCRL-JC-150051
}

IA WAINCE

IVLRMIOAE

WATIONAL

LABCAMTOAY

\section{Defining the Envelope for Sonic IR: Detection Limits and Damage Limits}

W. O. Miller, I. M. Darnell, M. W. Burke, C. L. Robbins

February 3, 2003

Thermosense XXV

Orlando, Florida, April 21-25, 2003 
This document was prepared as an account of work sponsored by an agency of the United States Government. Neither the United States Government nor the University of California nor any of their employees, makes any warranty, express or implied, or assumes any legal liability or responsibility for the accuracy, completeness, or usefulness of any information, apparatus, product, or process disclosed, or represents that its use would not infringe privately owned rights. Reference herein to any specific commercial product, process, or service by trade name, trademark, manufacturer, or otherwise, does not necessarily constitute or imply its endorsement, recommendation, or favoring by the United States Government or the University of California. The views and opinions of authors expressed herein do not necessarily state or reflect those of the United States Government or the University of California, and shall not be used for advertising or product endorsement purposes.

This work was performed under the auspices of the U.S. Department of Energy by University of California, Lawrence Livermore National Laboratory under Contract W-7405-Eng-48. 


\title{
Defining the envelope for sonic IR: detection limits and damage limits
}

\author{
Wayne O. Miller, Ian M. Darnell, Michael W. Burke, Christopher L. Robbins \\ Lawrence Livermore National Laboratory, 7000 East Ave., Livermore, CA 94550
}

\begin{abstract}
Work is presented which begins to define the envelope of applicability for the sonic IR NDE technique. Detection limits define the faintest flaw signal that can be perceived, which is a function of flaw size and depth, excitation strength and duration, and the detector limits (spatial, temporal, thermal). A unique contribution of the present work is the development of a model to predict the dynamic frictional heating of a crack, and this is combined with a transient heat transfer analysis to define the detection limits. Damage limits consider the risk of damage to a part from the application of the dynamic excitation. Experience has shown that the dynamic excitation can damage parts, notably for brittle materials such as ceramics with existing flaws. Since sonic IR is intended to be nondestructive it is important to test parts in a manner consistent with preserving the part integrity. The evaluation of damage limits assumes that additional part damage during testing is a fatigue process that propagates existing cracks. Paris' law for fatigue damage is employed to provide an estimate of fatigue crack propagation during the dynamic forcing. Both detection limits and damage limits are combined to create an envelope of applicability for sonic IR. Further experimental effort is required to tune and validate the analytical tools presented herein. This work was performed under the auspices of the U.S. Department of Energy by Lawrence Livermore National Laboratory under Contract W-7405-Eng-48.
\end{abstract}

Keywords: nondestructive evaluation, infrared imaging, heat transfer, dynamics, fatigue

\section{INTRODUCTION}

The sonic IR method for non-destructive evaluation (NDE) has seen rapid acceptance and progress in recent years. The method is fast, full field, relatively inexpensive and is suitable for a wide range of materials and part geometries. Our group at the Lawrence Livermore National Laboratory views sonic IR as a useful adjunct to more traditional NDE technologies, and we have spent the last three years developing a sonic IR capability and learning how best to apply it. We came to the early conclusion that even though sonic IR was obviously successful in many applications, the physics of the process were poorly understood and we set out to redress that lack of understanding. We are still pursuing that goal, but have achieved a reasonable level of understanding that appears to address all of the major physical phenomena.

One gauge of physical understanding is the ability to apply that knowledge in a quantitative and predictive manner. In particular we should be able to predict the IR signature produced by a particular flaw during a hypothetical sonic IR test, giving an indication as to whether or not the flaw can be detected. In addition we should be able to predict the likelihood of damaging a part due to excessive stress caused by the dynamic forcing used in sonic IR. We have seen many brittle ceramic parts fail during testing and so know first hand that sonic IR is not always non-destructive. 
We can define the envelope of applicability for sonic IR with such a predictive capability, and the work presented herein is our initial attempt at that task. The envelope of applicability defines the range of parts, flaws, and experimental parameters for which both (1) a particular flaw can be detected by an IR camera, and (2) the part will not be excessively damaged by the test. The first requirement is what we use to define the detection limit. We seek to define a priori the smallest and deepest flaws that can be detected by a proposed sonic IR experiment. If we know that the flaws we seek cannot be detected by a proposed sonic IR test, then we can possibly adjust the experimental parameters to bring a flaw above the detection limit or save the useless effort of trying to find them if no such adjustments are effective. Thus knowledge of the detection limits gives the tester a gauge on the chance of successful detection, and may suggest ways to improve that chance of success. The second requirement defines damage limits. For the same proposed sonic IR test we seek to know a priori if the testing procedure will damage the part beyond an acceptable level. Acceptable damage may be defined by the functional strength and life requirements of the part. If the proposed test will exceed the acceptable damage criterion then either the test should be modified to yield less damage or should not be performed. A proposed test is in the envelope of applicability for sonic IR if the predicted signal strength is above the detection limit and the predicted damage is below the damage limit, and this is the desirable situation for successful testing.

The physics of a sonic IR test are complex and cover a wide range of interacting topics. In broad strokes, the physics of interest are the dynamic response of the part to the forcing, the generation mechanism of heat at a flaw, the conduction of heat away from the flaw, the detection of the IR signal, and the damage mechanism. We have addressed each of these issues in the development given below. We have incorporated the simplest physical models available that appear to address each physics component. Nonetheless the determination of the envelope of applicability still requires some effort to assemble and analyze the different physical phenomenon for each unique situation. We have yet to validate the work presented herein with experimental data, and we expect that some assumptions and models will be modified as we do validate the work, although the basic framework should stand.

\section{DETECTION LIMITS}

The limits of detection define the faintest thermal signature from a flaw that can be detected by the sonic IR technique. To define these limits we need to predict the thermal signature from a flaw of interest. The prediction of the thermal signal will suffice to determine if the sonic IR method is capable of identifying flaws of interest.

Several factors are important in defining the thermal signal strength from a flaw. Consider first the issues of generating heat at a flaw. It is assumed herein that the heat is generated by friction at the flaw, which is induced by interfacial motion resulting from the dynamic forcing ${ }^{\mathrm{A}}$. Thus we have the forcing, the dynamic response, the crack type and the frictional model as important concerns. For our work, the forcing is by an ultrasonic welding horn in contact with the piece under test. We assume this forcing can adequately be described as a sinusoidal displacement boundary condition with an amplitude on the order of tens of microns, and a frequency on the order of tens of kilohert ${ }^{B}$. The dynamic response of the part depends on the material elastic properties, the part geometry and on the constraints used to fixture the part during forcing. As stated this is a general problem of elasticity and requires unique solutions for specific parts. We have incorporated a simplified finite element model to evaluate the dynamic behavior as described later. Finally the friction model evaluates the transduction of dynamic motion into thermal power. The true frictional behavior at a flaw is undoubtedly a complex issue that deserves advanced study to consider such effects as nonlinear dynamics, irregular

\footnotetext{
${ }^{\text {A }}$ Other mechanisms for producing heat from motion, such as plastic work, are possible but have not been evaluated.

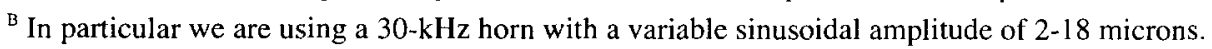


surfaces and non-planar geometry. However to move our efforts forward we have assumed planar flaws and a simple Coulomb friction model.

Next consider the issues of heat transfer. The total thermal energy produced depends on the dynamic response just described and on the duration of forcing. The flaw size determines the area of heat generation, so that minute flaws will produce vanishingly small thermal power while larger flaws will produce proportionately more power. The flaw depth affects the conduction of heat to the surface imaged by the IR camera. Hence surface flaws will appear warmer and sooner as the heat does not diffuse much before being imaged, while deep flaws appear cooler and later as the heat diffuses throughout the material. The material thermal properties affect the diffusion of heat. A low thermal diffusivity will allow heat to build up locally and spread slowly while a high thermal diffusivity will result in a weak and fleeting thermal transient. All of these factors have been incorporated into a transient heat transfer analysis for a generic heatgenerating flaw as described below.

Finally there are the issues of camera detection of the IR signal. The IR signal must radiate from the part surface to the camera. The quality and strength of that radiated signal depend on the temperature rise, the surface emissivity and on the background thermal noise. The camera must then detect the signal well above the noise floor of the camera, which defines the minimum detectable temperature rise. The camera must also be capable of imaging on the spatial and temporal scales that define the IR signal. The spatial scale is on the order of the flaw size while the temporal scale depends on the forcing duration ${ }^{\mathrm{C}}$ and on the decay transient. We have not formally incorporated the issues of camera detection into this work, but rely on intuitive consideration of these effects.

\subsection{Dynamic heating model}

The dynamic heating model predicts the amount of thermal power generated by a flaw during dynamic forcing. The model consists of two primary components; (1) a general theoretical model of frictional work at a flaw in a sinusoidally varying stress field, and (2) a simplified finite element model to predict the stress field. The stress field at any desired point in the part predicted by the finite element model is used as an input parameter for the theoretical heat generation model, which in turn yields the thermal power produced at the crack.

The development of the frictional work model is beyond the scope of this paper ${ }^{\mathrm{D}}$, although the basic assumptions can be described. The model assumes a planar crack undergoing cyclic loading. The crack is buried in a $3 \mathrm{D}$ homogeneous linear elastic body of arbitrary shape. A Coulomb frictional model is assumed, which generates work when there is both relative sliding of the two mating faces of the planar flaw, and a normal force pressing the faces together. The model allows a stick/slip sliding behavior. The model is most accurate when the flaw is much smaller than the body so that the flaw does not affect the homogeneous stress field. Further the model is most accurate when the flaw is either deeply buried in the body or is a surface crack perpendicular to the surface. The model loses accuracy for shallow buried flaws not perpendicular to the surface, although an error analysis has not been completed and we assume that the model is approximately valid for all flaws.

\footnotetext{
C The thermal response may be made steady state by continuous application of the dynamic forcing, although this can damage both the part under study and the forcing device.

D A detailed paper on the frictional work model is under review for future publication. Contact the authors for more detail.
} 
The results of the friction work model can be stated as shown in Eq. (1). Here, $g$ is the average frictional (thermal) power generated by the flaw, with typical units of $\mathrm{W} / \mathrm{m}^{2}$. The flaw has area $A$ and a typical dimension $\boldsymbol{s}$, and the dynamic forcing frequency is $\omega$. The typical dimension is usually the depth of a surface crack or half the length of an embedded crack. The friction coefficient is $\mu$, and the elastic modulus is $\boldsymbol{E}$. The shear and normal stress values in the plane of the flaw are $\sigma^{\tau}$ and $\sigma^{\mathrm{n}}$, respectively. The crack geometry (disk, half disk, etc.) is characterized by the nondimensional parameter $\boldsymbol{C}_{\mathrm{C}}$, which has a typical range of 2-8. The non-dimensional parameter $\boldsymbol{C}_{\mathrm{R}}$ is the stress ratio coefficient, and depends on the local stresses and the friction coefficient as shown in Eq. (2).

$$
\begin{aligned}
& g=C_{C} C_{R}\left(\frac{\sigma^{\tau} \sigma^{n} \mu s^{3} \omega}{E A}\right) \\
& C_{R}=\frac{1}{2}\left(1-\frac{\sigma^{n} \mu}{\sigma^{\tau}}\right)\left(1+\frac{1-\frac{\sigma^{n} \mu}{\sigma^{\tau}}}{1+\frac{\sigma^{n} \mu}{\sigma^{\tau}}}\right)
\end{aligned}
$$

A simplified finite element model is used to predict the stress values necessary to evaluate the frictional work model of Eq. (1). This finite element model solves the periodically steady-state elasticity problem for the specific part geometry, constraints and periodic forcing of interest, and so must be built for each unique case. No transient analysis is required as the solution is periodically steady-state, consequently the numerical solution is rapid and efficient. The finite element model is simplified in that no flaws need to be explicitly modeled; the finite element model represents the undamaged part. This is consistent with the frictional work model assumption that the flaw does not affect the homogenous stress field. A major advantage of this simplification is that a single finite element solution can be interrogated many times to evaluate flaws at any point and orientation in the body, which is a feature we exploit for probabilistic forecasts as described next.

\subsection{Probability of heat generation}

With the dynamic heating model just described, the thermal power generated by a specific flaw at a specific location and orientation can be evaluated readily. However, it is possible that a flaw could be at a location and orientation where it would fail to produce frictional work, and would hence be undetectable by sonic IR. The flaw orientation is important as the shear and normal stresses in the plane of the flaw will vary as the flaw is rotated within the homogeneous stress field. As a result of using the Coulomb friction model, there will be orientations that produce zero work and hence no thermal signature. Such is the case when the flaw is in a "clapping" mode with no shear stresses or in a pure sliding mode with no normal stresses. Work is produced at other intermediate orientations. The flaw location is important as the periodically steady-state solution will show peaks, valleys and nodes of stress and motion. Flaws located near nodes will show little tendency to produce thermal work at any orientation, while flaws near peaks and valleys will tend to produce maximum work.

In practice it is unlikely that the flaw location and orientation are known, as by the nature of NDE one is hunting for unknown flaws. Fortunately it is a simple extension of the dynamic heating model to effectively evaluate all flaw locations and orientations in a part at once. The results of this procedure can be viewed as a probability map of heat 
generation in the part, where the probability of a flaw generating a threshold amount of thermal power, $\boldsymbol{g}_{\text {MIN }}$, is determined at each point. The analyst can set $\boldsymbol{g}_{M I N}$ to a value that will generate the weakest detectable IR signal, using the heat transfer model described below and a knowledge of the IR camera sensitivity. The probability map then yields information on where a given flaw may or may not be detected with a comfortable level of certainty. Note that the same part can yield different detection probability maps depending on the constraints and forcing used in the finite element analysis, as these affect the dynamics of the part. Thus if one set of constraints and forcing produces a low probability of detection in a critical region, then changing the constraints and forcing location may yield a more favorable outcome.

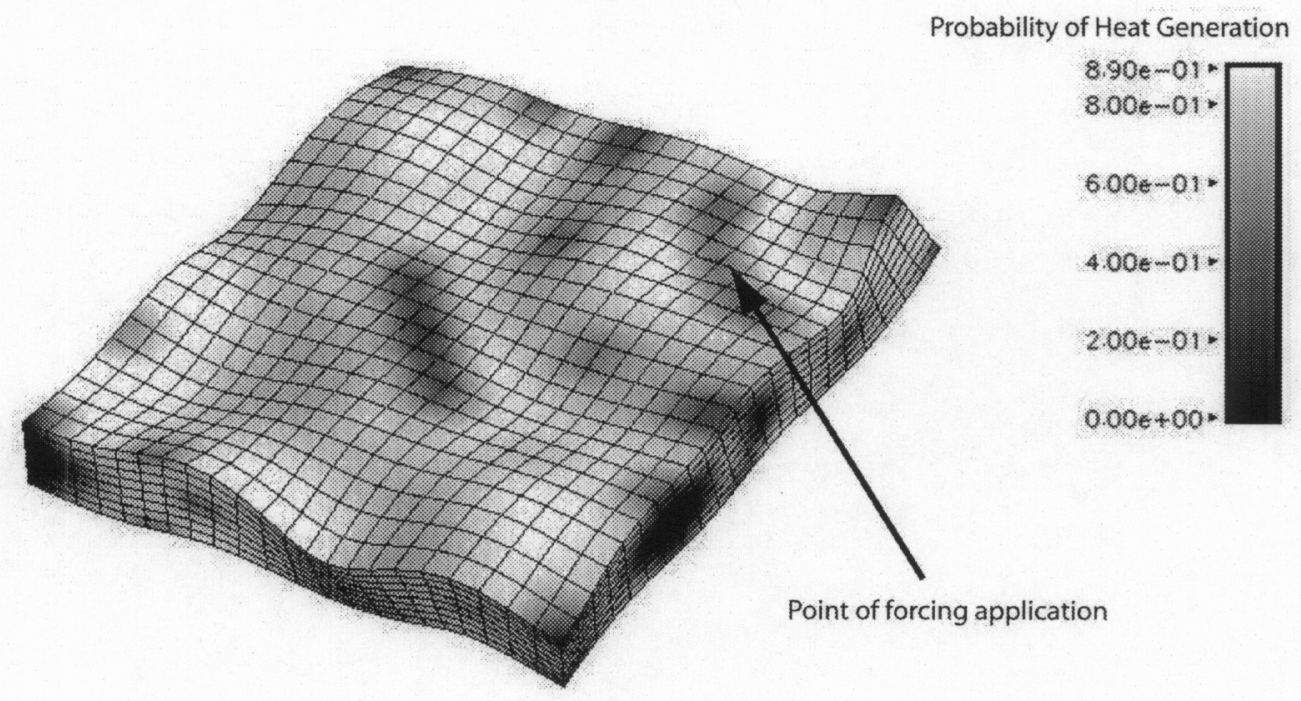

Figure 1: Heat generation probability map for a $2.5-\mathrm{mm}$ radius "half penny" surface crack producing $100 \mathrm{~kW} / \mathrm{m}^{2}$ of thermal flux.

Generating the probability map is accomplished by evaluating the frictional work model for a specific crack geometry at many orientations for each element in the finite element model. Each element has the local solution of the homogenous stress field available. Many orientations are used to statistically evaluate the variation of frictional work with orientation at each point. Combined, this process gives a complete detection probability distribution throughout the part for the specific constraints and forcing used in the finite element analysis. The probability solution is a post-processing step, and is rapid as the frictional work model is computationally simple to evaluate. An example of such a probability map is shown in Figure 1. The map shows the probability of a "half penny" surface crack with a radius of $2.5 \mathrm{~mm}$ producing $g_{M I N}=100 \mathrm{~kW} / \mathrm{m}^{2}$. The part is a 6" x 6" x 3/8" block of 7075-T6 aluminum with periodic forcing at $30 \mathrm{kHz}$ with an amplitude of 3 microns applied at the point indicated in the figure. The block is supported on a negligibly soft elastic foundation. The deformed shape represents the (greatly magnified) dynamic response of the part. The results indicate that over most of the surface, there is a probability of $60 \%$ or greater that a surface crack will produce at least 100 $\mathrm{kW} / \mathrm{m}^{2}$. There are isolated regions with much lower probability.

\subsection{Heat transfer model}

The heat transfer model completes the required detection limits physics by predicting the surface temperature on a part given the flaw size and depth, and the thermal power $g$ predicted by the dynamic heating model just described. We assume that the minimum detectable temperature rise is known based primarily on the camera sensitivity, and that the 
predicted temperature rise is compared against the minimum detectable temperature rise to evaluate whether a flaw can be detected or not. Conversely, the heat transfer model can be tuned to yield a detectable temperature for a given flaw by varying $g$, and this required power output is then be used to define the probability maps just described.

The heat transfer model predicts the transient temperature rise on the surface above a disk-shaped flaw of radius $\boldsymbol{R}$ that is parallel to the surface, and at depth $\boldsymbol{a}$ below the surface (Figure 2). The flaw is embedded in a semi-infinite cylindrical domain of depth $0<z<\infty$ and radius $r<\infty$. The flaw generates heat with a uniform surface flux $g$ for $0<t<t_{\theta}$, where $t_{0}$ is the duration of the dynamic forcing.

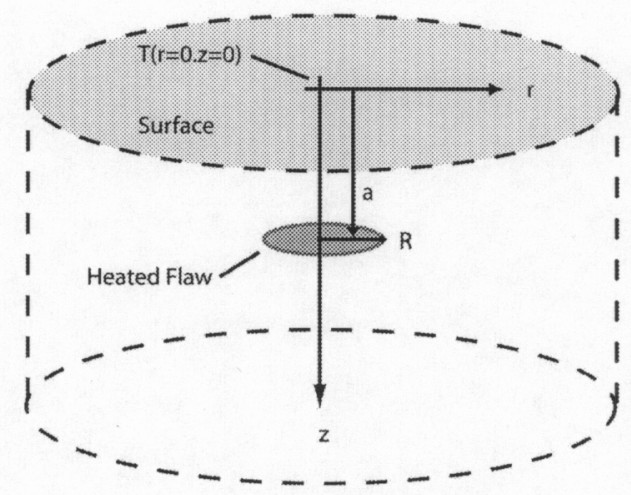

Figure 2: Geometry of the heat transfer model

The exact solution for this heat transfer problem was found for the point on the surface directly above the flaw center $(r=0, z=0)$ and for all times after the cessation heat input $\left(t>t_{0}\right)$. The point $(r=0, z=0)$ will show the maximum temperature rise of all surface points, and this maximum temperature will occur for $t>t_{0}$, so these solution restrictions do not invalidate the analysis. The solution thus obtained is given in non-dimensional form in Eqs. (3)-(5). The four non-dimensional parameters are the temperature group $\Theta=\boldsymbol{T} \boldsymbol{k} /(\boldsymbol{g} \boldsymbol{R})$ where $\boldsymbol{T}$ is temperature and $\boldsymbol{k}$ is thermal conductivity, the depth ratio $L=a / R$, the time ratio $\tau=t / t_{0}$, and the Fourier number $F o=\alpha t_{0} / R^{2}$, where $\alpha$ is the thermal diffusivity.

The non-dimensional form of Eq. (3) allows the solution to be presented as a set of generalized charts that can be interrogated for any desired part or flaw. The data is four-dimensional, corresponding to the groups $\{\Theta, \boldsymbol{L}, \tau, \boldsymbol{F o}\}$, and for visualization only variations in three dimensions can be plotted. We have chosen to plot variations in $\{\Theta, L, \tau$, , while holding $\boldsymbol{F o}$ constant for each plot, but varying $\boldsymbol{F} \boldsymbol{o}$ by plot over a useful range. Of course one could generate targeted plots for exact conditions if desired, removing the necessity to graphically interpolate the solution. The solution of Eq. (3) can have large gradients of $\Theta$ near $\tau=0$, which make the charts difficult to read. This problem is overcome by plotting $\ln [\Theta]$, rather than $\Theta$, as the solution amplitude.

$$
\begin{gathered}
\Theta\left(r=0, z=0, t>t_{0}\right)=\frac{\mathrm{I} 1-\mathrm{I} 2}{\sqrt{\pi}} \\
\mathrm{I} 1=2 e^{\frac{-L^{2}}{4 \tau F o}} \sqrt{\tau F o}-2 e^{\frac{-L^{2}}{4(\tau-1) F o}} \sqrt{(\tau-1) F o}+L \sqrt{\pi}\left(\operatorname{erf}\left[\frac{L}{2 \sqrt{\tau F o}}\right]-\operatorname{erf}\left[\frac{L}{2 \sqrt{(\tau-1) F o}}\right]\right)
\end{gathered}
$$




$$
\mathrm{I} 2=2 e^{\frac{-\left(L^{2}+1\right)}{4 \tau F o}} \sqrt{\tau F o}-2 e^{\frac{-\left(L^{2}+1\right)}{4(\tau-1) F o}} \sqrt{(\tau-1) F o}+\sqrt{L^{2}+1} \sqrt{\pi}\left(\operatorname{erf}\left[\frac{\sqrt{L^{2}+1}}{2 \sqrt{\tau F o}}\right]-\operatorname{erf}\left[\frac{\sqrt{L^{2}+1}}{2 \sqrt{(\tau-1) F o}}\right]\right)
$$
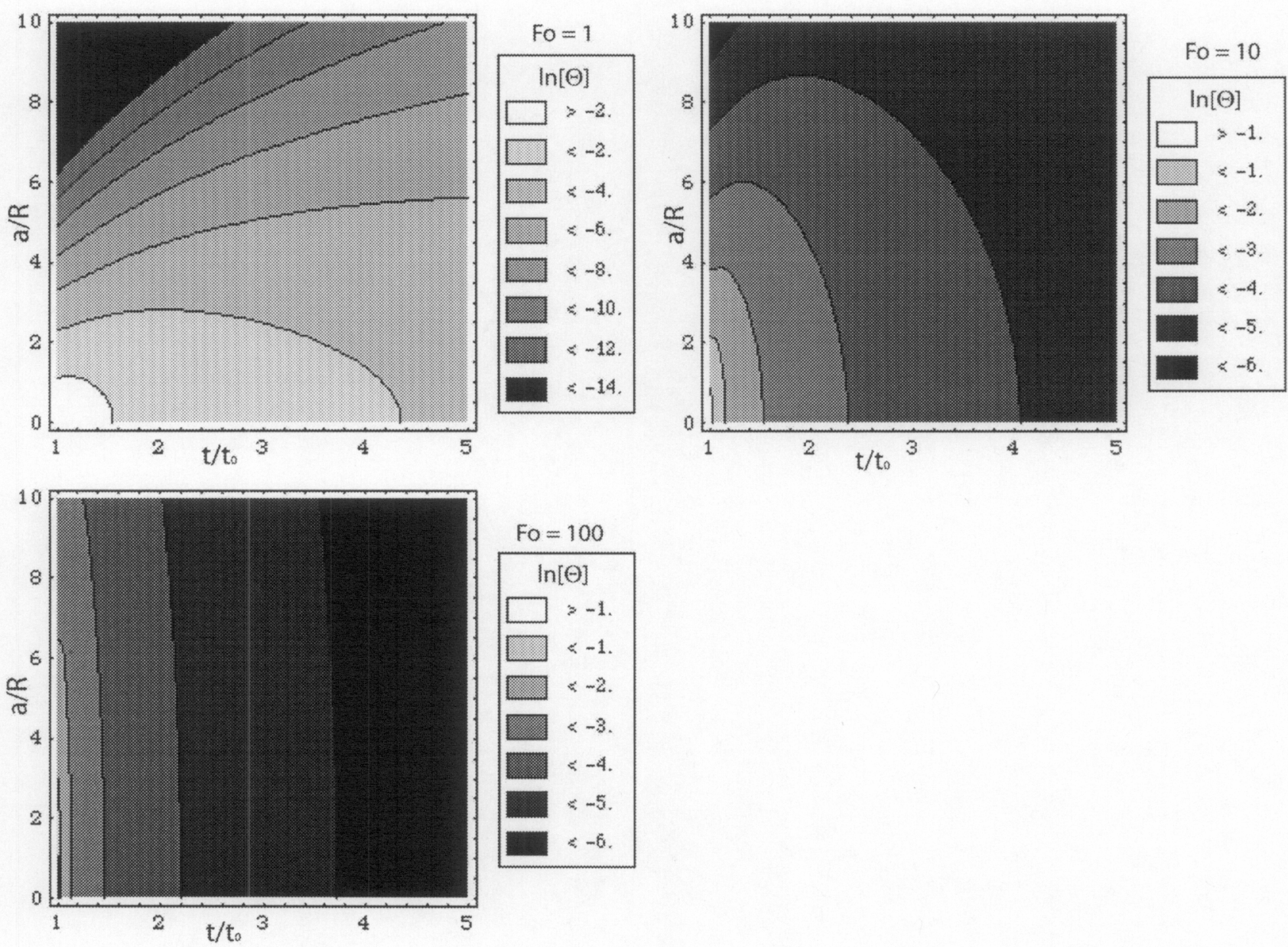

Figure 3. Non-dimensional solution of the heat transfer model at $\mathrm{Fo}=\{1,10,100\}$.

Examples of the generalized charts of the solution of Eq. (3) are shown in Figure 3 for $\boldsymbol{F o}=\{1,10,100\}$. The contours are elongated along the time axis for small $\boldsymbol{F o}$ and along the depth axis for large $\boldsymbol{F o}$. There are two complimentary observations of this behavior. First, cases with small $\boldsymbol{F o}$ have long-duration thermal signatures after the forcing has ceased, while cases with large $\boldsymbol{F} \boldsymbol{o}$ have fleeting thermal signatures after the forcing has ceased. Recalling the definition $\boldsymbol{F o}=\alpha \boldsymbol{t}_{0} / \boldsymbol{R}^{2}$, this suggests that long-duration thermal signatures can be obtained by (1) decreasing the thermal diffusivity $\alpha$, which impedes the conduction of heat away from the crack, (2) increasing the flaw size $\boldsymbol{R}$, which results in more energy deposition per unit time and favorably reduces the depth ratio $\boldsymbol{a} / \boldsymbol{R}$, and (3) reducing the forcing duration $\boldsymbol{t}_{\boldsymbol{\theta}}$, which reduces the temperature gradient (as well as the temperature rise), but also unfavorably increases the time ratio $t / t_{\theta}$. Note that varying $\boldsymbol{F o}$ does not define the absolute temperature rise, but only the non-dimensional behavior of the solution. 
The second observation is that cases with small $\boldsymbol{F o}$ favor surface flaws, while cases with large $\boldsymbol{F o}$ can identify deeper flaws. This time the interplay of variables is reversed as we seek to increase $\boldsymbol{F o}$ : (1) increasing the thermal diffusivity allows deeper heat generation to rapidly advance to the surface, (2) increasing the forcing time delivers more energy to the deep flaw thereby increasing the local temperature gradient, and also favorably reduces the time ratio $t / t_{0}$, and (3) decreasing the flaw size increases $\boldsymbol{F o}$, although this unfavorably increases the apparent depth $\boldsymbol{a} / \boldsymbol{R}$, and so is counterproductive.

These observations are mutually exclusive in terms of their desirability. On one hand, small $\boldsymbol{F o}$ provides long-duration transients, but reduces the strength of deep flaw signals. On the other hand, large Fo will help detect deeper flaws, but decreases the time available to detect the signals. In practice the range of $\boldsymbol{F o}$ will be determined by the case at hand, and this helps define the detection limits for a particular case as described next.

\subsection{Defining detection limits}

Up to this point we have developed a forward predictive capability in that we start with a known flaw, determine the heat generation and then the thermal signature. The definition of the detection limits is in effect a reverse predictive process in that we know the required thermal signature for detection based on our imaging sensitivity, and work backwards to define the envelope of flaw parameters that can produce thermal signatures of sufficient strength to be imaged. This reverse process is not necessarily unique, although the steps presented here should serve as a general guide.

Let us narrow the scope of the problem by assembling the known information. First and foremost, our imaging sensitivity will dictate a minimum detectable temperature rise, $\mathbf{T}_{\text {MIN }}$. We will use this as the threshold temperature for detection. Next, we will have a particular material, so the elastic properties and thermal properties are also known. We will also assume a known friction coefficient, although this may be considered a tunable parameter. These may well be all of the known values we can depend on as we move forward (or backward in essence) toward defining the detection limits.

Next we make the practical assumption that we seek a flaw greater than some minimum size $\boldsymbol{R}_{M I N}$, where $\boldsymbol{R}_{M I N}$ might be based on component lifetime arguments such as strength or fatigue life. With this assumption of a known flaw size we can proceed directly to define the detection limits envelope for that flaw size. If flaws of different sizes are considered, each will have it's own detection envelope. Thus a complete analysis of detection limits could result in determining detection limits for a variety of flaw sizes.

We can now employ the analytical tools just developed. To begin, we have complete information to use the dynamic heating model for a flaw of dimension $\boldsymbol{s}_{M I N}=\boldsymbol{R}_{M I N}$. The resulting maps of heat generation probability can be used to pick an appropriate value of $\boldsymbol{g}$ to use in the heat transfer model. If the range of $\boldsymbol{g}$ is large over the region of interest, then several detection limit analyses may be required in order to span the full range.

Finally the generalized charts from the heat transfer model are employed. We can put a lower bound on the thermal solution $\Theta$ as shown in Eq. (6), where the thermal power $g$ is that just derived from the dynamic heating model. The generalized solution charts are reviewed to isolate the contour envelope where $\ln [\Theta]>\ln \left[\Theta_{\mathrm{MIN}}\right]$, and this envelope defines the desired detection limits. All values of the non-dimensional crack parameters $\tau, \boldsymbol{L}$ and $\boldsymbol{F o}$ whose coordinates 
lie within this contour envelope are detectable in theory. In practical terms this leaves the forcing duration $t_{\theta}$ as the single free variable for the analysis. Varying $t_{0}$ simultaneously shifts both the time ratio $\tau$ and the Fourier number $\boldsymbol{F o}$.

$$
\Theta_{M I N}=\frac{T_{M I N} k}{g R_{M I N}}
$$

As an example, we will continue with the aluminum block of Figure 1, with a proposed 2.5-mm radius "half-penny" surface crack that is perpendicular to the surface. Surface cracks have a unique feature in our framework. The crack model is a half-circle perpendicular to the surface, while the heat transfer model assumes a heated disk that would lie on the surface $(a=0)$ for a surface crack. Thus there is a geometric mismatch in that the two models assume different shapes (half circle, full circle) and they are also orthogonal to each other. For deeply buried flaws this mismatch is not an issue as we can ignore local thermal effects due to orientation, and we make the surface areas equal. However for surface flaws, local effects are important as we seek to know the temperature precisely at a point in the middle of the half-circle crack where it intersects the surface. As it turns out, a symmetry argument shows that we can exactly predict the temperature at the center of the half-circle crack perpendicular to the surface (the crack model) using a full-circle heated disk of the same radius on the surface (the heat transfer model). This special-case symmetry argument requires that the heat transfer model use precisely half of the thermal flux $g$ predicted for the surface crack, but only for surface cracks. For buried cracks the full flux is used in the heat transfer model.

To proceed, we will assume that the surface crack generates $g=100 \mathrm{~kW} / \mathrm{m}^{2}$, which has a $60 \%$ or better chance as shown in Figure 1. We will use half of that, $50 \mathrm{~kW} / \mathrm{m}^{2}$, for Eq (6) for the special case of a surface crack. Let $T_{M I N}=0.01{ }^{\circ} \mathrm{C}$ be our IR threshold of detection. We have $\boldsymbol{R}_{M I N}=2.5 \mathrm{~mm}$ for this proposed crack and $k=160 \mathrm{~W} /\left(\mathrm{m}^{\circ} \mathrm{C}\right)$ for this aluminum. Using these values in Eq. (6) gives $\Theta_{M I N}=0.013$, or $\ln \left[\Theta_{M I N}\right]=-4.4$ which we use for the heat transfer graphs of Figure 3. In this special case of a surface crack, we set the depth ratio $a / \boldsymbol{R}=0$ in Figure 3, whereas we would normally seek solutions at all depth ratios. We interpolate between contours of -4 and -5 to find that the surface crack is detectable for the full time ratio range shown at $F o=1$, for $t / t_{0}<3$ at $F o=10$ and for $t / t_{0}<1.8$ at $\boldsymbol{F o}=100$.

\section{DAMAGE LIMITS}

The dynamic forcing used for sonic IR inspections has the potential to damage the part under test. Damage limits provide an a priori estimate of the potential for damage from a specific forcing condition, and also of the likely extent of the damage. The forcing condition is defined by the elasticity problem as previously discussed for the dynamic heating model. The main issues are the forcing amplitude and duration, the location of the forcing on the part, and the part itself (including constraints). These yield a unique solution to the periodically varying stress field in the part. Damage will result if the maximum stresses locally exceed a failure criterion, such as yield stress or fracture stress. Thus defining the damage limits requires that we first determine the stresses in a part, and then compare them against a suitable failure criterion. We have already accomplished the first task, as the stresses were determined using the simplified finite element model used in the dynamic heating model.

The failure method we assume herein is fatigue damage. This assumption is justified by our experience, which shows that sites with existing cracks or evidence of prior damage are most likely to show additional damage during sonic IR testing. To proceed, we hypothesize the existence of an initial crack of length $a_{l}$, and then determine the likely fatigue 
growth, $\Delta a$, of that crack during the dynamic forcing ${ }^{\mathrm{E}}$. The analyst must determine reasonable thresholds for both for the assumed initial crack size and for the allowable growth. For a part with no apparent damage, the initial crack length could be defined as the threshold of detection by available NDE methodologies. For a part with obvious damage, the initial crack length can be measured directly. A likely consideration in this determination is the allowable final crack size, $a_{F}=a_{l}+\Delta a$, which may be dictated by the functional strength or life requirements of the part.

The crack growth can be minimized by reducing the forcing amplitude, which reduces the cyclic stress, and by reducing the forcing duration $t_{b}$, which reduces the count of fatigue cycles, $N$. However these changes may weaken the IR signal from any true flaws in the part. Thus a balance must be found between IR detection strength and the likelihood of part damage. It is therefore desirable to know both the detection limits and the damage limits for each case of interest, and to tailor the NDE experiment and expectations of discovery appropriately.

\footnotetext{
E The length $\boldsymbol{a}$ is usually interpreted as the full crack length for surface cracks and the half-crack length for embedded cracks.
} 


\subsection{Fatigue crack growth}

Fatigue crack propagation is governed by different mechanics depending upon the life stage of the crack. These stages are illustrated in Figure 4 as regimes A, B and C. The crack growth per cycle is $\mathrm{d} a / \mathrm{d} N$, and the stress intensity range is $\Delta \boldsymbol{K}=\boldsymbol{K}_{\text {MAX }}-\boldsymbol{K}_{\text {MIN }^{*}}$ The stress intensity factor can be expressed as $\boldsymbol{K}=\mathbf{f}\left(\sigma, \boldsymbol{a}^{1 / 2}\right)$, where the functional form depends on the crack geometry. Thus $\Delta K=\mathrm{f}\left(\Delta \sigma, \boldsymbol{a}^{1 / 2}\right)$, which depends on the cyclic stress amplitude and the crack length.

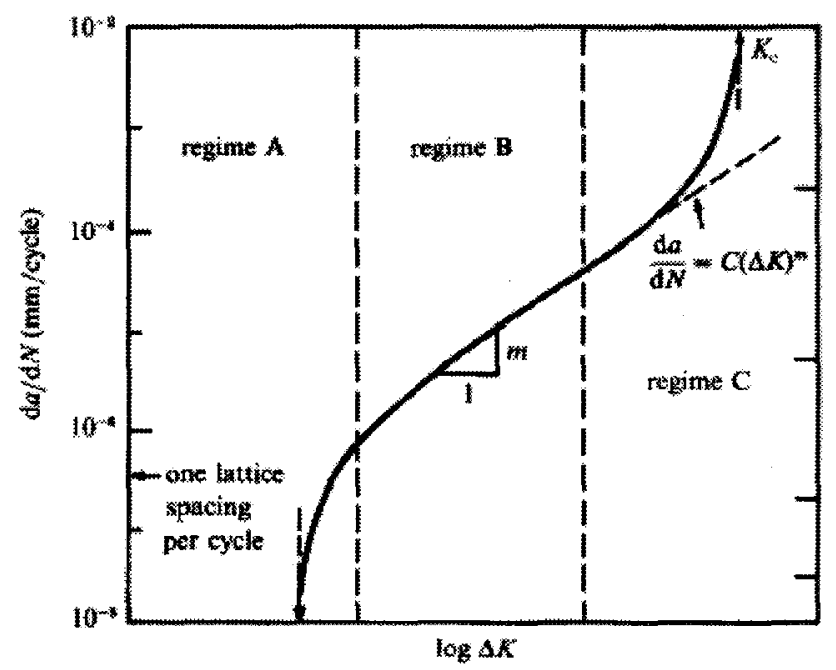

Figure 4. Fatigue crack propagation behavior. Paris' law holds in regime B.

Regime $\mathrm{A}$ is the crack initiation stage where small cracks are stationary or grow slowly. Regime $\mathrm{C}$ is the last, rapid growth stage for large cracks, which ends in material failure when the stress intensity factor reaches the static limit, $\boldsymbol{K}_{\boldsymbol{C}}$. Regime $\mathrm{B}$ typically defines the working life of a fatigue crack, and propagation is described by a power law relating growth rate to stress intensity range. This is Paris' law of fatigue crack growth ${ }^{1}$, which is shown in Eq. (7). The parameters $\boldsymbol{C}$ and $\boldsymbol{m}$ are material properties that are found by experiment and are available in the literature for many materials. Table 1 lists the values for a few materials of interest ${ }^{2}$. Within the Paris regime the stress intensity range can be expressed as shown in Eq. (8). This shows that the stress intensity range increases as the square root of the crack length. Hence small cracks grow more slowly than large cracks for a given stress range. The parameter $\boldsymbol{Y}$ depends on the crack geometry. For the two-dimensional case, a center crack has a value of $Y=1.00$ while for an edge crack the value is 1.12 . The value for a three-dimensional surface or center crack will be somewhat lower than for the twodimensional case.

$$
\begin{aligned}
& \frac{d a}{d N}=C \Delta K^{m} \\
& \Delta K=Y \Delta \sigma \sqrt{\pi a}
\end{aligned}
$$


Table 1. Paris' law fatigue properties for common materials (stress in MPa, length in $\mathrm{m}$ ).

\begin{tabular}{|c|c|c|}
\hline & $\boldsymbol{C}$ & $\boldsymbol{m}$ \\
\hline Aluminum, 7075-T6 & $1.1 \times 10^{-11}$ & 3.9 \\
\hline Titanium, Pure & $6.8 \times 10^{-12}$ & 4.4 \\
\hline Steel, 18/8 & $3.2 \times 10^{-12}$ & 3.1 \\
\hline
\end{tabular}

\subsection{Fatigue damage model}

We have chosen to model fatigue crack growth using Paris' law. Paris' law is valid in regime B of Figure 4, the main working life of the crack, and also provides an analytically simple model of crack growth. More sophisticated and universally applicable fatigue models are available, and could be incorporated into the damage model if needed to improve damage predictions.

Paris' law is used with the simplified finite element model to predict fatigue crack growth. The finite element model evaluates the periodic stress field $\Delta \sigma$ at all locations in the part. We will conservatively assume that the crack is oriented with regard to the local principal stresses so that $\Delta \sigma$ is maximized, thereby enhancing the growth rate. This occurs when the crack is orthogonal to the principal stress with the greatest absolute value, and this stress defines $\Delta \sigma$. Similarly the geometry parameter $\boldsymbol{Y}$ can be conservatively chosen as the maximum value in order to maximize crack growth. Finally Paris' law can be integrated over the desired number of cycles to predict the crack growth $\Delta a$. Evaluation of Paris' law is embedded as a post-processing operation for the finite element model, similar to the friction work model of Eq. (1). The results can be presented as a surface map of crack growth, such as shown in Figure 5. This is the same example case of Figure 1. The geometry parameter $\boldsymbol{Y}$ has a value of 1.0 for this "half-penny" surface crack perpendicular to the surface. In this example, a $2.5-\mathrm{mm}$ radius surface crack is predicted to increase in radius between $28 \mathrm{~nm}$ and $133 \mu \mathrm{m}$ after 10,000 fatigue cycles, depending on location.

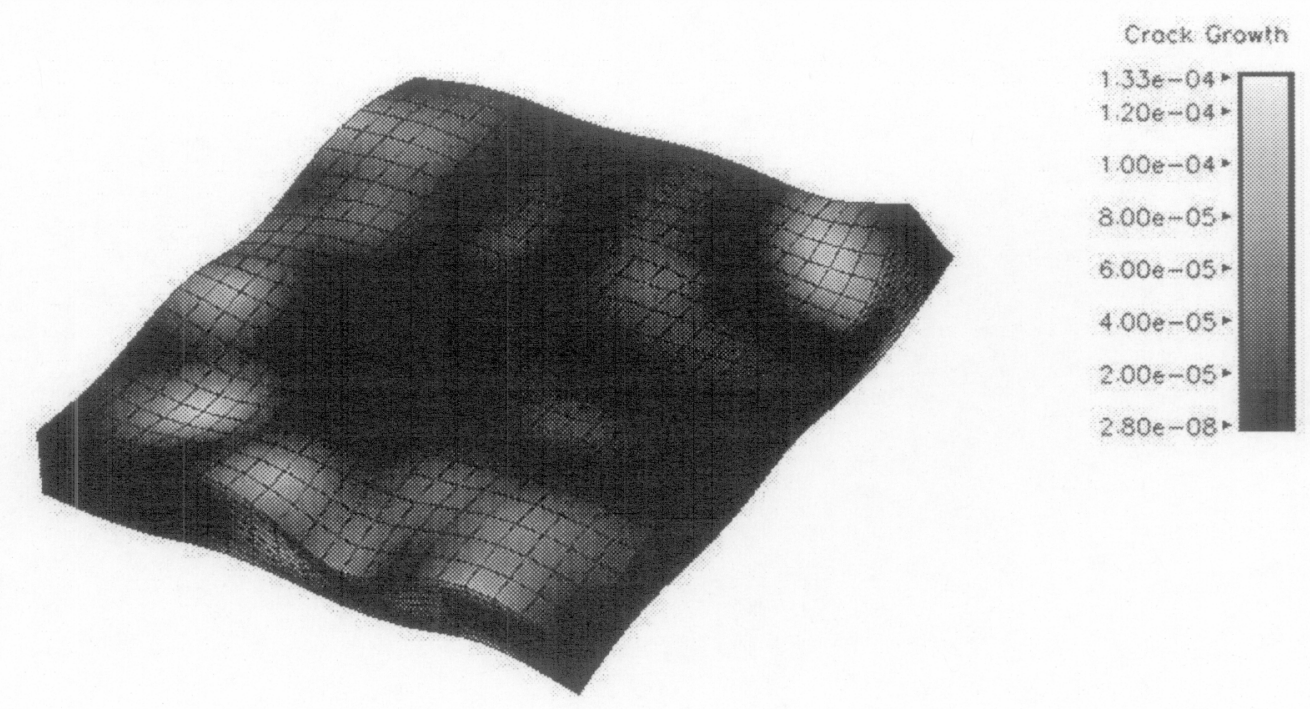

Figure 5. Crack growth map for a 2.5-mm radius "half penny" surface crack after 10,000 fatigue cycles (scale units in meters). 


\section{SUMMARY}

We have developed a predictive methodology for both detection limits and damage limits of sonic IR NDE testing. Detection limits give estimates on the smallest and deepest flaws that can be detected for a particular part. Damage limits estimate the potential for fatigue damage from the cyclic loading of the part. When combined, these limits define the envelope of applicability of sonic IR.

Detection limits are derived from predictions of the transient surface temperature on a part arising from a flaw heated by the dynamic forcing. The flaw is assumed to generate heat by friction arising from the dynamic motion of the part, as defined by an analytical friction work model. The dynamic motion of the part is predicted by a simplified finite element model that does not explicitly include any flaws. The amount of heat generation at a flaw depends on both the location of the flaw and on the local orientation of the flaw within the part, which results in an overwhelming quantity of data. As a result the heat generation predictions are presented in terms of the probability of the heat generation exceeding a specified threshold at each point, and this is viewed as a probability map on the part. A transient heat transfer model that uses the heat generation rate predicted by the friction work model, and a specified duration of forcing, determines maximum surface temperatures. The surface temperatures are compared to the minimum detectable temperature of the IR system to define the detection limits. The envelope of detection limits includes the range of flaw parameters that can produce a sufficiently strong thermal response for IR detection. These flaw parameters include flaw size, depth below the surface, and forcing duration. This envelope of detectability can be graphically represented as contours on the generalized non-dimensional solution of the heat transfer model.

Damage limits predict the possibility and extent of damage to a part undergoing dynamic forcing. In particular we assume fatigue crack propagation that can be described by Paris' law. An existing crack of specified length is hypothesized to exist in the part at an unknown location, where the initial length could be chosen as the threshold of detection for an undamaged specimen. The stress amplitude necessary for crack propagation is immediately available from the simplified finite element model used for the detection limits. Crack growth can be conservatively estimated at any point in the body by assuming the crack is orientated to maximize the stress amplitude. At each point in the part, Paris' law is integrated over the number of forcing cycles to determine the crack growth during a sonic IR test. This results in an estimate of crack growth at each point in the part that can be viewed as a surface map. The final crack length can be compared to an acceptance criterion based on part functional requirements such as strength or remaining lifetime. If the final crack length exceeds the acceptance criterion then the probability of damage is high and a less damaging set of test parameters should be chosen.

The methodology outlined in this paper has not yet been tuned and proven by direct experimental observation, and this is a critical step that remains before these tools can be reliably used. We have initially chosen simplified models of the physics in order to build the complete analytical framework. These simplified physics include the Coulomb friction model for heat generation, the simplified finite element model for dynamic response, the two-dimensional heat transfer model for surface temperature predictions and the Paris fatigue model for damage predictions. Future enhancements of the methodology will include more sophisticated physical models, as experience dictates.

\section{REFERENCES}


1. Paris, P. and Erdogan, F., "A critical analysis of crack propagation laws," Journal of Basic Engineering, Transactions of the American Society of Mechanical Engineers, December 1963, pp. 528-534.

2. Hellan, K., Introduction to Fracture Mechanics, McGraw Hill, Inc., 1984, p. 154. 